\title{
The Cardiac Genetics Clinic: a model for multidisciplinary genomic medicine
}

Dominica Zentner MB BS(Hons), FRACP, PhD

Tina N Thompson

Paul A James MB ChB, DPhil, FRACP

Alison Trainer MB ChB, MSc, PhD

Lesley C Adès $M B$ BS, FRACP, MD

Ivan Macciocca BSc, MHSc(GenCouns) FHGSA

Jessica A Taylor BSc, MGenCouns

Kirsty Mann

BSc, MGenCouns

Michael Bogwitz BSc(Hons) GradDipGenCouns, PhD

Nigel Lewis MB ChB, MRCP, CCDS

Natalie Morgan BNurs, GradDip(GenCouns)

Jitendra Vohra MD, FRACP, FRCP

Ingrid Winship MD, FRACP, FACD

1 Royal Melbourne Hospital Melbourne, VIC.

2 Children's Hospital a Westmead, Sydney, NSW.

3 Murdoch Childrens Research Institute, Melbourne, VIC

4 Victorian Institute of

Forensic Medicine,

Melbourne, VIC

5 Office for Research

Melbourne Health

Melbourne, VIC

dominica.zentner@ mh.org.au

doi: 10.5694/mjal4.01674

Podcast with Dr Dominica Zentner and Dr Paul James available at www.mja.com.

au/multimedia/podcasts nherited heart disease can be well

managed by preventive strategies

if detected early. Building on an expanding body of literature on the contribution of hereditary heart disease to sudden cardiac death (SCD) ${ }^{1-3}$ and the well-validated principles of predictive gene testing in other single-gene disorders, the Cardiac Genetics Clinic (CGC) was formally established at the Royal Melbourne Hospital in 2007. Published data have supported the benefit of clinical screening in such clinics. ${ }^{4}$ However, detection of a causative mutation, where possible, also allows identification of individuals who are currently clinically unaffected.

The CGC embodies a multidisciplinary model for translating research into international best-practice care. ${ }^{5}$ This model exemplifies the translation of genetics to genomics in practice, and also aims to educate and inform individuals, allowing them to assume responsibility for their own ongoing care and health.

The CGC is a joint undertaking by the clinical genetics and cardiology units at the Royal Melbourne Hospital. It is managed by a cardiac trained nurse who performs telephone intake on all referrals, and as well as coordinating screening tests and collating relevant clinical information on individuals and their family members before the clinical appointment. ${ }^{6}$ On average, patients wait 4 to 5 months between referral and appointment. Cardiologists, clinical geneticists and genetic counsellors, as well as fellows and trainees in each field, attend the clinic. Patients may attend individually or with family members; families of deceased patients generally attend as a family unit. Clinics are preceded by a multidisciplinary planning meeting, allowing discussion and decision-making as a group - a particularly important step in making equitable and consistent decisions regarding access to clinic-funded genetic testing and evidence-based medical advice.

\section{Abstract}

Objectives: To describe patient characteristics, standard operating procedure, and uptake of genetic testing at the multidisciplinary Cardiac Genetics Clinic (CGC) at the Royal Melbourne Hospital during its first 6 years.

Design: Database exploration of referral diagnoses, sex, number of clinic visits and incidence of genetic testing in a population of individuals attending the CGC.

Setting: Tertiary referral hospital (Royal Melbourne Hospital) providing cardiac genetics services to the state of Victoria.

Participants: All individuals initially attending the clinic between July 2007 and July 2013, either as the proband or as an at-risk family member.

Main outcome measures: Classification of patients into diagnostic categories, number of probands and at-risk relatives assessed, incidence and outcomes of genetic testing.

Results: 1170 individuals were seen for the first time over the 6-year period; $57.5 \%$ made only one visit. The median age was 39 years. Most were encompassed within four broad diagnostic categories: cardiomyopathy (315 patients), aortopathy (303 patients), arrhythmia disorders (203 patients) and resuscitated cardiac arrest and/or family history of sudden cardiac death (341 patients); eight patients had "other" diagnoses. Genetic testing (mutation detection or predictive testing) was undertaken in 381 individuals $(32.6 \%)$, and a pathogenic mutation was identified in $47.6 \%$ of tests, representing $15.3 \%$ of the total population.

Conclusion: The CGC fulfils an important role in assisting clinicians and patients by reviewing genetic cardiac diagnoses. Clinical practice during the study period moved from a selected candidate gene approach to broader gene panel-based testing. This move to next-generation sequencing may increase the detection of mutations and variants of unknown significance. A major contribution by the clinic to the care of these individuals and their families is the provision (or negating) of a diagnosis, and of a plan for managing risks of predictable cardiac disease.

The referral base includes general practitioners, physicians and cardiologists; self-referrals are also possible. Relatives of individuals who have died unexpectedly or with autopsy findings suggestive of a genetic cardiac condition are referred by the forensic pathologists at the Victorian Institute of Forensic Medicine(VIFM).

As a consultative service, the patient is discharged back to the referring clinician once the genetic component has been resolved, or an appropriate management plan is drawn up for the referral of at-risk individuals.

Data are maintained in a customised relational database that links individuals from the same family. In recognition of the complexity of genetics and current knowledge limitations in cases where a genetic contribution is suspected but unconfirmed, a plan for periodic file review is prepared, with an electronic reminder system associated with the database. Further, when the clinical implications of a detected genetic variation are unclear, the results are added to the database for future review and reclassification should their interpretation change.

This article presents details of our clinic's processes and patient population as we move from disease-specific gene testing to next generation sequencing (NGS).

\section{Clinic structure}

Our clinic employs a standard operating procedure (Box 1). We have 


\section{Standard operating procedure of the Cardiac Genetics Clinic Referral \\ Clinic appointment day \\ After the appointment \\ Follow-up correspondence with patient and family \\ Coordination of additional special investigations and collation of results \\ Follow-up as required (return to clinic if genetic testing ordered; review of individual in cases of clinical uncertainty; discharge back to referring doctor, or assist with referral to appropriate service; discharge from need for care) \\ Database notation if future file review is planned, including responsible person and interval}

formalised the process of patient review across the departments of genetics and cardiology in our hospital. As many patients travel long distances to visit us, we attempt to provide same-day cardiac testing, before the clinical review. Additional specialised cardiac testing, such as a flecainide test or adrenaline challenge, are provided after the clinical review.

\section{Methods}

We extracted data on all patients $(n=$ 1170) who had a first appointment at the CGC between July 2007 and July 2013. This data set included both the proband (the person who triggered the referral to the CGC) and their atrisk relatives. In families where referral followed a death, we describe only the outcomes of the at-risk relatives reviewed.

We extracted the following details for each patient from the CGC database: referral phenotype; number of probands referred (all individuals within this time period were identified as either a proband or an at-risk relative); age at referral; sex and number of at-risk relatives who were screened. Clinical phenotypes are presented in broad diagnostic groups for the purposes of this article.

Approval for the data analysis was provided by the Melbourne Health Human Research Ethics Committee (QA2014096).

\section{Results}

Of the individuals seen, 359 (30.7\%) were probands, $331(28.3 \%)$ were at-risk family members, and 480 $(41.0 \%)$ were at-risk family members referred to the CGC following the death of a family member.

\section{Referral diagnosis for individuals seen at the Cardiac Genetics clinic}

The distribution of diagnostic categories at referral is presented in Box 2 .
Inherited cardiac disease in our clinic is categorised into four broad groups: cardiomyopathies, aortopathies, arrhythmias and survivors of cardiac arrest together with families of an SCD individual. In this study:

- Cardiomyopathy $(n=315)$ included dilated, hypertrophic, restrictive and arrhythmogenic ventricular cardiomyopathies.

- Aortopathy $(n=303)$ included Marfan syndrome, Loeys-Dietz syndrome, familial aortic aneurysm and dissection syndrome, and connective tissue disorders.

- Arrhythmia disorders $(n=203)$ included the long-QT, short-QT, Brugada, catecholaminergic polymorphic ventricular tachycardia, mitral valve prolapse, atrial fibrillation, Wolf-ParkinsonWhite, and sick sinus syndromes.

- We grouped individuals and families seen after a resuscitated cardiac arrest or SCD $(n=341)$.

A small number of patients with other diagnoses $(n=8)$ were seen at the CGC during the study period.

\section{Number of visits}

The number of visits per individual during the study period ranged from one to five. Most patients (57.5\%)

2 Reason for referral of the 1170 individuals seen at the Cardiac Genetics Clinic over a 6-year period

$\square$ Cardiomyopathy
$\square$ Aortopathy
$\square$ Arrhythmia
$\square$ Sudden cardiac death
$\square$ Other

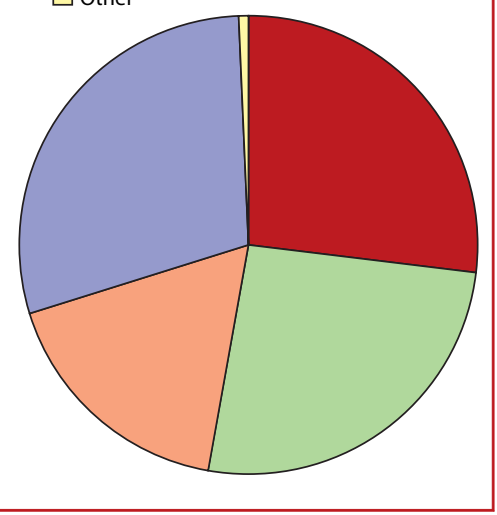




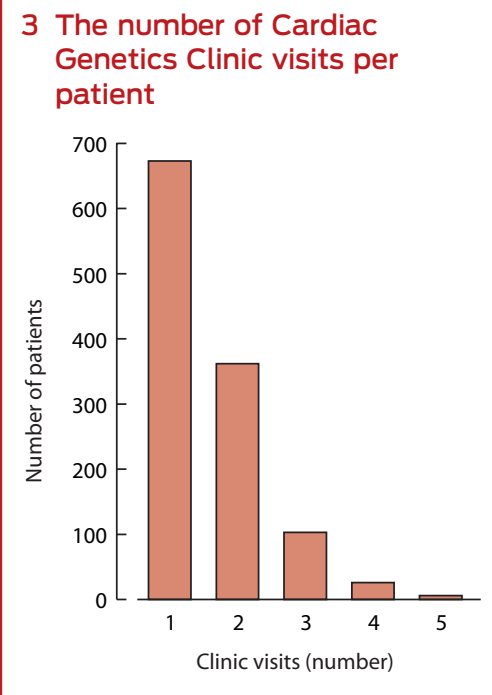

were seen only once. A very small number of individuals (six) were seen on five occasions (Box 3 ).

Sex

Slightly more women (603) were seen at the CGC than men (565), but the difference was not statistically significant $\left(\chi^{2}\right.$ test; $\left.P=0.28\right)$.

Age

The median age of the study population at the time of patient review was 39 years (range, 13-93 years). Although we operate an adult service, 51 teenagers between 13 and 17 years of age (median age, 16 years) were seen while accompanying family members for a family appointment. Younger teenagers had been offered appointments at a paediatric service, but chose to come to the Royal Melbourne Hospital to avoid being separated from their families. The distribution according to sex and age in each broad diagnostic category is presented in Box 4 . There was no statistically significant difference in age between the genders for each diagnostic category (MannWhitney) or in the gender spread within each disease category ( $\chi^{2}$ test).

\section{Genetic testing}

Genetic testing was undertaken in 381 individuals ( $32.6 \%$ of population; median age, 38 years; range, 14-93

\begin{tabular}{|c|c|c|c|c|}
\hline \multirow[b]{2}{*}{ Diagnosis } & \multicolumn{2}{|c|}{ Women } & \multicolumn{2}{|c|}{ Men } \\
\hline & Number & $\begin{array}{c}\text { Median age, } \\
\text { years (range) }\end{array}$ & Number & $\begin{array}{c}\text { Median age, } \\
\text { years (range) }\end{array}$ \\
\hline Cardiomyopathy & 165 & $40(14-93)$ & 150 & $40(14-76)$ \\
\hline Aortopathy & 143 & $38(14-73)$ & 160 & 35 (15-71) \\
\hline Arrhythmia disorders & 105 & $40(15-72)$ & 98 & $38(14-85)$ \\
\hline $\begin{array}{l}\text { SCD or resuscitated } \\
\text { cardiac arrest }\end{array}$ & 188 & $40(13-82)$ & 153 & $36(14-82)$ \\
\hline Other & 3 & $44(41-56)$ & 5 & $40(21-56)$ \\
\hline
\end{tabular}

years). Of these, five individuals had undergone a total of eight previous genetic tests at another service; the results of these tests were available to us and are included in our summary below. The 788 patients not tested were of similar age (median, 39 years; range, $13-82$ years). In 11 individuals (median age, 31 years; range, 15-61 years), a karyotype or microarray test was also ordered, but the results of these tests are not included in this article. Only eight of those who underwent genetic testing were 70 years of age or older $(<0.1 \%$ of those tested). The genetic tests undertaken were of two types: mutation detection (in 170 individuals, $44.6 \%$ ) and predictive genetic tests (in 211 individuals, 55.4\%). In total, $47.4 \%$ of probands and $26.0 \%$ of atrisk family members were offered genetic testing. The apparently low number of at-risk family members offered testing highlights the fact that it has not been the usual practice of the clinic to undertake genetic testing in the absence of a clinical phenotype. For this reason, genetic testing was not undertaken in at-risk relatives when genetic testing of a proband was either inconclusive or uninformative, or when no clinical phenotype had been established for a deceased proband or in an at-risk relative. Of the 211 at-risk family members who underwent genetic testing, this resulted in a new diagnosis for 58 otherwise healthy individuals (27.5\% of those tested).

The frequency and type of genetic testing performed in each of the broad diagnostic categories is presented in Box 5. It is notable that our current clinical practice has not expanded to routinely include molecular autopsy in all cases of unexplained death, but is restricted to situations when a diagnosis is suggested by the results of clinical screening in relatives.

The 376 individuals who underwent genetic testing initiated by our service may have had a single gene or a number of genes screened during a single genetic testing episode. In $93.0 \%$ of patients, only one genetic testing episode occurred. In 22 individuals, two genetic testing episodes were undertaken; three individuals had three episodes, and one individual had four. A total of 407 genetic testing episodes were undertaken by the clinic during the 6-year period. Pathogenic mutations were detected in 179 individuals $(47.6 \%$ of those tested), or $15.3 \%$ of all patients reviewed by the clinic. These figures underestimate the total number of genetic tests ordered by the clinic, as testing of subsequently deceased probands was not captured in the current data, as previously described.?

\section{Discussion}

The CGC aims to confirm or negate a suspected diagnosis of an inherited cardiac condition to allow implementation of a personalised management plan for the affected individuals and their family members. Attending individuals undergo appropriate screening investigations and examination, and genetic testing is offered 
5 The number and percentage of individuals in each family diagnosis category who underwent genetic testing (mutation detection or predictive testing)

\begin{tabular}{lcccc} 
& Cardiomyopathy & Aortopathy & $\begin{array}{c}\text { Arrhythmia } \\
\text { disorders }\end{array}$ & $\begin{array}{c}\text { SCD or resuscitated } \\
\text { cardiac death }\end{array}$ \\
\hline Total number of patients & 315 & 303 & 203 & 341 \\
Genetic tests (\% of diagnostic category) & $154(48.9 \%)$ & $101(33.3 \%)^{*}$ & $97(47.8 \%)^{\dagger}$ & $29(8.5 \%)$ \\
Median age of tested patients, years (range) & $40(15-93)$ & $30(14-67)$ & $42(18-85)$ & $40(18-62)$ \\
Mutation detection tests (\% of all tests) & $51(33 \%)$ & $65(64.4 \%)$ & $38(39 \%)$ & $16(55 \%)$ \\
Number of positive results (\% of tests) & $32(63 \%)$ & $33(51 \%)$ & $20(53 \%)$ & $3(19 \%)$ \\
Predictive tests (\% of all tests) & $103(66.9 \%)$ & $36(35.6 \%)$ & $59(61 \%)$ & $13(45 \%)$ \\
Number of positive results (\% of tests) & $48(46.6 \%)$ & $10(28 \%)$ & $27(46 \%)$ & $6(45 \%)$ \\
Overall positive results (\% of all tests) & $80(51.9 \%)$ & $43(42.6 \%)$ & $47(48 \%)$ & $9(31 \%)$ \\
\hline
\end{tabular}

$\mathrm{SCD}=$ sudden cardiac death. * Four patients had been tested before their appointment with the Cardiac Genetics Clinic. $\dagger$ One patient had been tested before their appointment with the Cardiac Genetics Clinic. No genetic testing was undertaken in the eight patients with diagnoses outside the four broad categories.

when indicated, always accompanied by counselling and education. This allows for a targeted risk management strategy or release from screening, as appropriate. For mutation carriers, counselling includes discussion of reproductive options, including both prenatal diagnosis and pre-implantation genetic diagnosis. Clinic staff liaise widely with other specialists, particularly with paediatric cardiologists who provide the clinical care for younger members of identified at-risk families. Translational research is also a focus, and the clinic has been involved with patient groups in education and advocacy, when invited.

The effectiveness of the clinic is facilitated by a formal relationship with the VIFM, whose staff refer at-risk families after potentially genetic cardiac deaths. This includes communication regarding the likely need for genetic testing, which allows the timely storage of biological material. $^{5}$ To foster robust discussion about the relevance and interpretation of both post mortem findings and family evaluation, the CGC and VIFM meet on a quarterly basis to discuss cases of particular interest or clinical difficulty. This has proved to be critical in allowing detailed discussion of borderline pathological findings where the significance may not be fully appreciated or which could be incorrectly understood to suggest a particular diagnosis. ${ }^{8}$
The clinical benefit achieved by the simultaneous review of patients by a cardiologist and a clinical geneticist includes the identification of rare diseases and accurate assessment of the utility of genetic testing. This is borne out by the high yield of mutation detection by genetic testing, which highlights the importance of a multidisciplinary clinic and the usefulness of the whole-family approach. ${ }^{9}$

Published data on genetic testing as part of standard clinical practice of cardiovascular disease in large cohorts is limited, in contrast to genetic testing in a research setting. A Dutch analysis of the results of genetic testing in 6944 individuals identified potential disease-causing mutations in a third of the families seen. The greatest yield was in families with long-QT syndrome (47\%) and hypertrophic cardiomyopathy $(46 \%),{ }^{10}$ similar to our clinical testing results.

The ultimate intention of the CGC is to prevent adverse cardiac events through early identification and optimal management advice to atrisk individuals. There are currently no long-term data that show improved outcomes are achieved by this approach, and providing these data remains a long-term aspiration of our service. Similarly, although it is anticipated that cost-effectiveness can be achieved, largely by excluding from screening genotype-negative individuals from high-risk families, this remains to be confirmed.

Access to and the application of genetic testing in the CGC has evolved over time. Initially, an iterative approach was adopted, but this was subsequently replaced by small testing panels and, more recently, by a large gene panel (currently including up to 101 known cardiac disease genes). The use of broad panel testing commenced in the clinic during 2013, and the full impact of NGS technologies, in comparison with single mutation detection, has yet to become apparent. With increasing access to large gene panels comes the burden of interpreting multiple genetic abnormalities. ${ }^{11,12}$ This involves significantly increased time commitment for both the molecular genetics laboratory and for the clinical geneticists and genetic counsellors who inform the patients and their families. These challenges were discussed in a recent review that highlighted the importance of the relationship between the laboratory and clinicians in the delivery of genetic services. ${ }^{13}$ It is possible that the current targeted panel approach to testing is a transitionary phase before more comprehensive approaches, such as full exome sequencing, are introduced in the clinic. The current methodology has the advantage that, while it generates an increased volume of results that must be managed in the clinic, 
the risk of completely unanticipated results is minimised.

We anticipate that the use of genetic testing at the CGC will increase in the future, reflecting both its potentially decreasing cost as well as the increased utility of multiple gene testing that is now routine. The currently recommended care model for genetic medicine, ${ }^{9}$ achieved by a multidisciplinary team working together with the genetics laboratory, provides an effective means for translating advances in genomic medicine into clinical practice.
Acknowledgements: We thank the service provider laboratories in Australia, especially the Victorian Clinical Genetic Service, and overseas, from whom we were able to obtain accredited genetic testing results that could be used in a clinical setting.

Competing interests: No relevant disclosures.

(c) 2015 AMPCo Pty Ltd. Produced with Elsevier B.V. All rights reserved. 
1 Hofman N, Tan HL, Alders M, et al. Active cascade screening in primary inherited arrhythmia syndromes: does it lead to prophylactic treatment? J Am Coll Cardiol 2010; 55: 2570-2576.

2 Tester DJ, Ackerman MJ. The role of molecular autopsy in unexplained sudden cardiac death. Curr Opin Cardiol 2006; 21: 166-172.

3 van Langen IM, Hofman $\mathrm{N}$, Tan $\mathrm{HL}$, Wilde AA. Family and population strategies for screening and counselling of inherited cardiac arrhythmias. Ann Med 2004; 36 Suppl 1: 116-124.

4 Gimeno JR, Lacunza J, García-Alberola $A$, et al. Penetrance and risk profile in inherited cardiac diseases studied in a dedicated screening clinic. Am J Cardiol 2009; 104: 406-410.

5 Priori SG, Wilde AA, Horie $\mathrm{M}$ et al. Executive summary: HRS/EHRA/ APHRS expert consensus statement on the diagnosis and management of patients with inherited primary arrhythmia syndromes. Europace 2013; 15: 1389-1406.

6 Thompson T, Morgan N, Connell V, et al. The role of specialist nurses in cardiac genetics - the Victorian experience: supporting partnerships in care. Aust J Adv Nurs 2015 (in press).

7 Kumar S, Peters S, Thompson T, et al. Familial cardiological and targeted genetic evaluation: low yield in sudden unexplained death and high yield in unexplained cardiac arrest syndromes. Heart Rhythm 2013; 10: 1653-1660.

8 Papadakis M, Raju H, Behr ER, et al. Sudden cardiac death with autopsy findings of uncertain significance: potential for erroneous interpretation. Circ Arrhythm Electrophysiol 2013; 6: 588-596.
9 Ingles J, Zodgekar PR, Yeates L, et al. Guidelines for genetic testing of inherited cardiac disorders. Heart Lung Circ 2011; 20: 681-687.

10 Hofman N, Tan HL, Alders M, et al. Yield of molecular and clinical testing for arrhythmia syndromes. Report of 15 years' experience. Circulation 2013; 128: 1513-1521.

1 Ingles J, Semsarian C. Conveying a probabilistic genetic test result to families with an inherited heart disease. Heart Rhythm 2014; 11: 10731078.

12 Wilde AA, Behr ER. Genetic testing for inherited cardiac disease. Nat Rev Cardiol 2013; 10: 571-583.

13 Arndt AK, MacRae CA. Genetic testing in cardiovascular diseases. Curr Opin Cardiol 2014; 29: 235-240. 